International Journal of Canadian Studies

Revue internationale d'études canadiennes

\title{
Britannicité et multiculturalisme canadien
}

\section{Donald Ipperciel}

Numéro 45-46, 2012

Francophonies, Interculturality, Cultures and Strategies

Francophonies, interculturalité, cultures et stratégies

URI : https://id.erudit.org/iderudit/1009907ar

DOI : https://doi.org/10.7202/1009907ar

Aller au sommaire du numéro

\section{Éditeur(s)}

Conseil international d'études canadiennes

\section{ISSN}

1180-3991 (imprimé)

1923-5291 (numérique)

Découvrir la revue

Citer cet article

Ipperciel, D. (2012). Britannicité et multiculturalisme canadien. International Journal of Canadian Studies / Revue internationale d'études canadiennes, (45-46), 277-306. https://doi.org/10.7202/1009907ar
Résumé de l'article

Nous défendrons la thèse selon laquelle le multiculturalisme canadien puise sa source idéologique dans un multiculturalisme normatif déjà présent dans le Troisième Empire britannique, c'est-à-dire le " Commonwealth britannique ". En effet, l'idée de multiculturalisme représentait une position légitime à laquelle on avait recours dans l'espace discursif de cette époque. Nous croyons en fait que l'idée même de britannicité recelait à cette époque une notion normative de multiculturalisme. L'analyse débute par un bref historique du terme " britannique ", suivie par une présentation de la vision multiculturelle propre au Troisième Empire à travers trois intellectuels de cette époque: Alfred Zimmern, John Buchan (dit Lord Tweedsmuir) et Édouard Montpetit. 


\title{
Donald Ipperciel
}

\section{Britannicité et multiculturalisme canadien}

\section{Résumé}

Nous défendrons la thèse selon laquelle le multiculturalisme canadien puise sa source idéologique dans un multiculturalisme normatif déjà présent dans le Troisième Empire britannique, c'est-à-dire le « Commonwealth britannique ». En effet, l'idée de multiculturalisme représentait une position légitime à laquelle on avait recours dans l'espace discursif de cette époque. Nous croyons en fait que l'idée même de britannicité recelait à cette époque une notion normative de multiculturalisme. L'analyse débute par un bref historique du terme " britannique », suivie par une présentation de la vision multiculturelle propre au Troisième Empire à travers trois intellectuels de cette époque: Alfred Zimmern, John Buchan (dit Lord Tweedsmuir) et Édouard Montpetit.

\begin{abstract}
The author defends the thesis that Canadian multiculturalism draws its ideology from a normative multiculturalism already present in the Third British Empire, that is, the "British Commonwealth". In fact, the idea of multiculturalism was a legitimate position and one that we could look to in the discursive space of this era. We believe that, during this era, the very idea of Britishness concealed a normative notion of multiculturalism. Our analysis begins by a brief history of the term "British", followed by a presentation of the multicultural vision specific to the Third Empire from the standpoint of three intellectuals of this time: Alfred Zimmern, John Buchan (known as Lord Tweedsmuir) and Édouard Montpetit.
\end{abstract}

\section{Introduction}

Nous souhaitons par ce texte contribuer au débat sur les origines idéologiques du multiculturalisme canadien, ce qui, notons-le, est autre chose que d'analyser ses origines strictement historiques ${ }^{1}$, l'accent étant davantage placé sur les idées que sur les événements. Dans la mesure où cette question touche à la façon dont les Canadiens construisent leur identité nationale, que ce soit sur le mode négatif ${ }^{2}$ ou positif ${ }^{3}$, on comprendra à quel point elle peut $\mathrm{s}^{\prime}$ embrouiller dans un souk idéologique. En ce sens, le concept de multiculturalisme, qu'il soit canadien ou européen, prend racine dans un contexte politique plus large qui conditionne certaines attitudes axiologiques et normatives. En Europe, par exemple, le multiculturalisme est souvent compris comme réponse au problème d'intégration d'une population musulmane islamiste. C'est sur cette base qu'il est, souvent, fortement critiqué ( $c f$. Taguieff 2008; Hillebrand 2006). Au Canada, le multiculturalisme est diversement interprété selon les positions idéologico-politiques qui s'y rapportent. Ainsi, le multiculturalisme a été présenté comme une trouvaille de Pierre Elliot Trudeau, dont le dessein 
aurait été de confondre le nationalisme québécois ( $c f$. p. ex. Gagnon 2000), ou encore de « faire avaler le bilinguisme anglais-français aux minorités de l'Ouest » (Gagnon 2007); comme le fruit des revendications de communautés est-européennes (Resnick 2005, 57), de Paul Yuzyk en particulier, immortalisé par son célèbre discours au Sénat canadien sur le multiculturalisme à l'époque de John Diefenbaker (Yuzyk 1964); comme un rejeton du passé colonial où l'oppression passée de cultures non dominantes aurait donné lieu à des revendications culturelles (Sosoe 2002); et ainsi de suite.

En règle générale, les commentateurs se sont avant tout intéressés aux causes proximales du multiculturalisme canadien. Dans ce contexte, on a raison de ramener son origine aux suites de la Commission Laurendeau-Dunton et à Trudeau, ou encore indirectement au mouvement des droits civiques aux États-Unis. Même ceux qui veulent attribuer la paternité du multiculturalisme à Yuzyk $^{4}$ ne font que repousser son origine de quelques années. Nous aimerions pour notre part considérer une cause plus distale, à notre avis plus apte à saisir les mouvements de fond en jeu. Partant du principe que l'histoire ne fait pas de sauts, il y a lieu de postuler des circonstances préalables ayant favorisé l'émergence de législations et de politiques publiques sur le multiculturalisme durant les années 1970. Nous défendrons la thèse selon laquelle le multiculturalisme canadien puise sa source idéologique dans un multiculturalisme normatif déjà présent au Troisième Empire britannique, c'est-à-dire le « Commonwealth britannique $»^{5}$. Loin de nous l'idée de poser que le Canada aurait alors effectivement été un havre multiculturel. Suffisamment d'écrits ont au contraire démontré la prévalence de l'anglo-conformité dans les pratiques tant publiques que privées au Canada (cf. Kymlicka 2001, 28-29; 2003, 72, 119; Palmer, 1990). Il nous suffira ici d'établir que l'idée de multiculturalisme représentait une position légitime à laquelle on avait recours dans l'espace discursif de cette époque. Nous entendons le concept de multiculturalisme non pas en un sens descriptif, qui renvoie à une factualité démographique, ni au sens de politiques gouvernementales, telles qu'elles ont été mises en œuvre depuis les années 1970, ce qui cadrerait mal avec la perspective historique adoptée ici. De toute évidence, le multiculturalisme dont il est ici question est normatif, et renvoie à l'idéal de cohabitation des cultures à l'intérieur d'un même État et à la dimension identitaire qui en découle pour le projet de construction nationale. Il est pris en un sens large qui inclut aussi le « multiculturalisme multinational » et le «multiculturalisme polyethnique », pour reprendre la distinction d'Éric Breton $(2000,160)^{6}$.

Certes, on aurait raison de rappeler que la période d'entre-deux-guerres est elle-même préparée par le $19^{\mathrm{e}}$ siècle impérial, contexte où l'Empire britannique en particulier - est présenté comme modèle de cohabitation des peuples ${ }^{7}$. Lord Acton, on le sait, a été l'un des apôtres les plus éminents du principe impérial et de la diversité culturelle à l'époque victorienne. Mais une telle cohabitation des peuples présupposait, en contexte impérial, une 
puissance tutélaire en mesure d'inspirer les communautés « inférieures » à atteindre un progrès civilisationnel (Acton 1907). On pourrait alors parler de multiculturalisme hiérarchique où la culture hégémonique - en l'occurrence britannique - l'emporte sur toutes les autres. En revanche, dans la période d'entre-deux-guerres, les Britanniques affirment officiellement et solennellement pour la première fois - et cela influencera le plan normatif -, l'égalité formelle des nations en son sein ( $c f$. infra). Que l'on croie ou non en la bonne foi de cette déclaration, il n'en reste pas moins que la cohabitation des peuples et des cultures est présentée, à un niveau normatif, de façon plus explicitement égalitaire, bien que, dans le détail, comme on le notera plus loin, on y perçoive encore un fond de hiérarchisme. C'est en tout cas ce que nous chercherons à illustrer à l'aide de positions d'acteurs éminents de cette période. On aurait aussi raison de rappeler que le concept de britannicité a joué, au Canada, un rôle politique important dès le début du $19^{\mathrm{e}}$ siècle, notamment par l'entremise de la pensée de Pierre Bédard, mais aussi dans le mouvement des Patriotes. Cependant, ce que défendent Bédard et ses émules ne porte pas tant sur un idéal de multiculturalisme que sur une défense des droits libéraux (égalité politique, liberté d'expression, etc.) pour tous les sujets sur la base de la tradition britannique ( $c f$. Ajzenstat 1990; 1991). Certes, il s'agit de rétablir l'égalité des « races ", mais précisément en en faisant abstraction, non pas en les réhabilitant à l'intérieur d'une pensée politique. En d'autres mots, c'est l'esprit universaliste d'un Kant plutôt que le culturalisme de Herder qui anime sa pensée.

Nul ne peut douter qu'un vent nouveau s'était levé après la Première Guerre mondiale: on assiste alors à un nouvel essor du principe de l'autodétermination des petites nations, telle que popularisée par le président Wilson; à la création de la Société des Nations, fondée sur l'égalité des petites et des grandes nations; à la promulgation du Statut de Westminster instituant une communauté de nations libres et égales, un Commonwealth de «nations sœurs ». Voilà autant de signes de l'émergence du principe d'égale dignité des peuples et des cultures. C'est à cette époque aussi qu'apparaît la métaphore de la mosaïque pour décrire la société canadienne ( $c f$. Gibbon 1938, viii-ix; Day 2000, 149-153). Sa première occurrence chez Victoria Hayward (1922) prendra un sens plutôt descriptif. Mais chez Kate Foster (1926), la métaphore est utilisée clairement dans une perspective d'identité nationale et de nation-building. Enfin, en 1938 paraît la volumineuse étude de Murray Gibbon intitulée Canadian Mosaic, l'une des premières grandes célébrations du multiculturalisme canadien.Nous croyons que l'idée même de britannicité recelait à cette époque une notion normative de multiculturalisme. En ce sens, la britannicité peut être comprise comme précurseure du multiculturalisme canadien. C'est la raison pour laquelle nous commencerons l'analyse par un bref historique du terme " britannique ", question de préciser le sens que nous lui attribuerons dans la suite du texte. Nous nous emploierons ensuite à faire ressortir la vision multiculturelle propre 
au Troisième Empire à travers trois intellectuels de cette époque. Nous ferons d'abord référence aux travaux d'Alfred Zimmern qui, en tant que penseur idéaliste de l'Empire britannique, met particulièrement en relief sa visée normative. Le lien entre la pensée abstraite d'un penseur britannique et le multiculturalisme canadien d'entre-deux-guerres sera assuré par la présentation de la pensée du représentant de la Couronne au Canada et chef d'État à cette époque, soit le gouverneur général John Buchan. Finalement, afin de nous assurer que la pensée de la britannicité-comme-multiculturalisme ait pénétré de quelque façon des groupes autres que les Anglo-Saxons durant la période qui nous intéresse, nous aborderons le cas d'Édouard Montpetit, un nationaliste canadien-français, représentant, donc, d'une frange minoritaire de l'Empire britannique.

\section{Bref historique du terme « britannique »}

La naissance du terme « britannique » comme concept politique remonte à l'union formelle de l'Écosse et de l'Angleterre en 1707, suivant l'Acte d'Union. Le terme se présente d'emblée comme concept supérieur (un hyperonyme, diraient les linguistes) englobant les concepts désormais subalternes d' « anglais » et d' « écossais ». Or, contrairement à Linda Colley qui, auteure de l'important ouvrage Britons: Forging the Nation, 1707-1837, présuppose une certaine stabilité du concept jusqu'à son effritement récent ${ }^{8}$, il faut plutôt reconnaître dans l'histoire du terme un mouvement d'expansion et de contraction conforme aux vicissitudes de l'Empire.

En effet, par suite des Actes d'Union de 1800 associant le royaume d'Irlande à la Grande-Bretagne, le terme " britannique » en est venu à inclure également l'Irlande 9 , et ce, jusqu'en 1922, année où le traité anglo-irlandais entrait en vigueur, consacrant ainsi l'indépendance de la République d'Irlande. Selon John Pocock, cependant, ce serait la colonisation d'outre-mer qui aurait forgé un véritable sentiment de britannicité chez les Anglais, Écossais et Gallois (d'après McIntyre 2009, 70). Lors de l'apogée de l'Empire britannique durant la période victorienne, le concept de « Greater Britain » (Plus-Grande-Bretagne) a été introduit par Charles Wentworth Dilke, un politicien anglais d'allégeance libérale, spécialiste des questions coloniales. Selon ce dernier, « si deux petites îles se font par courtoisie appeler "Grande", l'Amérique, l'Australie et l'Inde doivent former une Plus-Grande-Bretagne » $(1869, \mathrm{x})$. Contrairement à ce que laisse sous-entendre ce passage, Dilke référait ici aux colonies pour autant qu'elles fussent anglophones ou gouvernées par les Anglais ${ }^{10}$. C'était là une vision commune en Angleterre, popularisée entre autres par John Robert Seeley dans son ouvrage maintes fois réédité Expansion of England $(1883 ;$; $f$. Armitage 2000, 16). Au Canada du 19e siècle, on sait par exemple que le terme " Canadien » était réservé aux Canadiens français, alors que les Canadiens anglais s'identifiaient pour la plupart comme des « Britanniques $»^{11}$. 
Puis, le terme s'est appliqué graduellement à tous les Blancs de l'Empire ${ }^{12}$, une restriction à laquelle Gandhi chercha à remédier en Afrique du Sud au tournant du siècle ( $c f$. Severence 1997, 33 sqq.). Enfin, à partir de 1914, la britannicité devenait formellement l'apanage de tous les sujets du roi. Avec la British Nationality and Status of Aliens Act de 1914, tous les individus nés dans les colonies étaient considérés comme britanniques, peu importe leur ethnie ou leur race. Même durant la Seconde Guerre mondiale, selon les directives du ministère de l'Information de la Grande-Bretagne, le terme « britannique » ne devait pas être utilisé lorsqu'on référait uniquement au Royaume-Uni. Le terme était réservé aux troupes provenant de l'Empire tout entier (McIntyre 2009, 67). Cet état de fait prit formellement fin en 1948 avec le British Nationality Act, mais effectivement par la maturation croissante de la conscience nationale des dominions ${ }^{13}$. Après la Seconde Guerre mondiale, la britannicité s'est repliée sur l'espace couvert par le Royaume-Uni, voire réduite à une identité résiduelle ${ }^{14}$. Paradoxalement, si l'on considère le passé raciste de l'Empire, mais sans doute en conséquence de l'extrême expansion de la britannicité dans la période d'entre-deux-guerres, les non-Blancs de la Grande-Bretagne se considèrent aujourd'hui plus volontiers comme « britanniques » que les Blancs qui, eux, se définissent davantage comme «Anglais », « Écossais 》 ou « Gallois » (Hughes 2009, 4).

Il semble donc que l'« exotique Empire d'outre-mer » n'ait pas toujours été perçu dans son altérité, comme le veut Linda Colley $(2003,7)$. Pour une courte période, l'Empire au-delà de la Grande-Bretagne avait été intégré dans un «nous » hétéroclite et inclusif. Cette courte période correspond aux années de l'entre-deux-guerres, où s'érigea le "Troisième Empire britannique ». En conséquence, malgré le caractère " infiniment insaisissable » du terme « britannique », pour reprendre le mot de David McIntyre (2009, 65), et les nombreuses confusions et contaminations avec le terme " anglais ", c'est en ce sens large, sanctionné par l'usage lors du Troisième Empire, que nous l'entendrons dans la suite de ce texte.

\section{Britannicité et multiculturalisme du Troisième Empire : Alfred Zimmern}

On distingue habituellement trois périodes dans l'histoire de l'Empire britannique. Les débuts du Premier Empire remontent à 1583, année où l'un des pionniers de la colonisation anglaise, Humphrey Gilbert, prend possession de Terre-Neuve au nom de la Couronne (Canny 1998, 34). Il prendra fin en 1776 ou en 1783, selon qu'on se réfère à la Déclaration d'indépendance des États-Unis ou à la conclusion de la Guerre d'indépendance par le Traité de Paris. La perte des treize colonies américaines marque le point de rupture et le passage au Second Empire (ibid., 92). Ce nouvel Empire sera fondé sur la puissance navale et le contrôle des voies maritimes. Il connaîtra son apogée durant la période victorienne et prendra fin avec la Première Guerre mondiale, alors que 
s'éveillent les consciences nationales et qu'un vent d'idéalisme wilsonien se fait sentir dans plusieurs régions du monde. On assiste alors à la naissance du Troisième Empire, aussi connu par la désignation «British Commonwealth of Nations », formule conçue par Arthur Balfour lors de la Conférence impériale de 1926 (Millat 2008, 124), popularisée par le général afrikaner Jan Smuts (Zimmern 1934, 3; McIntyre 2009, 33) et consacrée par le Statut de Westminster de 1931. Désormais, les États membres du Commonwealth, les dominions, sont considérés comme des « communautés autonomes au sein de l'Empire britannique, de statut égal, en aucune façon subordonnées l'une à l'autre eu égard à quelque aspect de leurs affaires intérieures et extérieures, bien qu'unies par une allégeance commune à la Couronne, et librement associées comme membres du Commonwealth britannique des nations » (Imperial Conference 1926, 1). Avec le Commonwealth des nations, l'Empire se fait désormais plus égalitaire. Il touchera cependant à sa fin avec la Seconde Guerre mondiale ${ }^{15}$.

Nous aimerions, dans cette section, porter notre attention sur l'une des premières grandes études consacrées au Troisième Empire ${ }^{16}$, rédigée pendant les années turbulentes de la formation de cette entité impériale, c'est-à-dire The Third British Empire d'Alfred Zimmern (1934). D'autres écrits sur ce thème ont aussi vu le jour durant la période d'entre-deux-guerres, mais ils portent principalement sur l'impact du Commonwealth britannique sur les relations internationales ou l'« ordre mondial », comme c'est le cas notamment de The British Commonwealth of Nations (1920) de H. Duncan Hall ou de The Conduct of British Empire Foreign Relations Since the Peace Settlement (1928) de Arnold Toynbee, par exemple ${ }^{17}$. L'ouvrage de Zimmern, bien qu'attentif à la question des relations internationales et de l'internationalisme, nous permet en outre, comme nous le verrons par la suite, une lecture du Commonwealth britannique qui met en relief son rôle dans le développement de l'idée normative de diversité ethnique et de multiculturalisme. C'est la raison pour laquelle nous nous y attarderons davantage.

The Third British Empire de Zimmern a d'abord été rédigé en 1926, année de la célèbre Conférence impériale ayant donné lieu à la Déclaration de Balfour, et réédité avec modifications en 1927 et en 1934. L'auteur était à son époque un grand spécialiste de relations internationales. Il a été le premier à occuper la chaire Woodrow Wilson en relations internationales à la University College of Wales, Aberystwyth, le premier professeur Montague Burton en Relations internationales à Oxford, et l'un des pères des Relations internationales comme discipline universitaire (Rich 2003, 79). S'inscrivant dans la mouvance idéaliste, pacifiste et internationaliste célèbre, il a été un architecte important de la Société des Nations et directeur adjoint de son Institut de coopération intellectuelle, précurseur de l'UNESCO ${ }^{18}$. En 1946, il a été sélectionné pour le prix Nobel de la paix. Zimmern voyait dans le Troisième Empire un modèle pour l'internationalisme. Il importe peu, ici, de considérer la pertinence de ses 
analyses empiriques de l'Empire; nous prendrons plutôt note de la dimension normative qu'il cherchait à faire valoir en lui.

Dans sa plus grande généralité, Zimmern présente l'Empire britannique dans une dialectique de l'unité et de la diversité. D'un côté, on retrouve une unité assurée par une communauté politique, symbolisée par la Couronne, ses idéaux et ses institutions. L'idéal directeur est celui de la liberté, tant individuelle, telle qu'elle est assurée par un État de droit protégeant les individus contre l'arbitraire ${ }^{19}$, que celle des peuples, représentée par le principe d'autodétermination. Si le principe de liberté a souvent été bafoué dans le passé de l'Empire, il n'en représente pas moins à ses yeux une norme à laquelle aspirait le Troisième Empire, laquelle se réalisait progressivement, selon lui, entre autres par l'établissement de dominions au sein de l'Empire. Zimmern compare l'Empire à une " procession » de communautés en route vers une complète autodétermination (Zimmern 1934, 8). Certes, le principe de tutelle (trusteeship) auquel adhéraient Zimmern et l'Empire représentait toujours une forme de paternalisme à l'égard des territoires administrés, mais l'histoire ultérieure confirmera que le dessein d'accorder aux peuples leur liberté formelle était effectivement sincère de la part de 1'Empire ${ }^{20}$. Ce mouvement vers une liberté toujours croissante aurait sa source, selon Zimmern, dès le début du Second Empire, comme le confirment l'Acte de Québec (cf.ibid., 180) et l'Acte constitutionnel de 1791. Ce dernier assurait que les droits constitutionnels britanniques ne soient « pas l'apanage et le privilège exclusifs des habitants de la Grande-Bretagne et de leurs descendants chez soi et d'outre-mer, mais qu'ils [reviennent] de droit à tous ceux vivant sous le drapeau britannique [...]» (Zimmern 1934, 25). Une telle conception de l'Empire britannique aura des conséquences sur l'idée même de britannicité. Selon Zimmern:

le contenu de l'adjectif "britannique" [...] se prête difficilement à une interprétation exacte. Ce n'est pas un adjectif de race, de nationalité ou de territoire. [...] Il dénote plutôt une tradition politique et sociale et un mode de vie dans lequel cette tradition se révèle. Il s'agit d'un mode de vie caractérisé par des habitudes communes, des institutions communes et une certaine philosophie tacite des affaires publiques. (Ibid., 96) 21

La britannicité se présente donc dès l'abord chez Zimmern comme concept politique et social.

À cette unité s'oppose, de l'autre côté, une diversité à plusieurs niveaux: géographique, puisque 1'Empire s'est étendu à tous les continents; raciale, avec des représentants de toutes les races; religieuse, incluant toutes les grandes religions; culturelle, dont les cultures germanique (les Afrikaners), latine (les Canadiens français) et slave (en particulier dans l'Ouest du Canada); et de gouvernement, pour autant que diffèrent les gouvernements du Royaume-Uni 
et d'Irlande, des dominions et des communautés dépendantes du gouvernement impérial, ces derniers constituant un groupe lui-même hétéroclite (cf.ibid., 6-11).

Dans la dialectique de l'unité et de la diversité, il y va avant tout de la façon dont l'unité peut effectivement s'accommoder de la diversité. En d'autres termes, comment la diversité peut-elle subsister sans exercer des forces purement centrifuges et autodestructrices? La réponse de Zimmern à cette question repose sur sa critique du nationalisme politique, qu'il qualifie de " mazzinisme » (cf. Rich 2003, 82). Il faut savoir que Zimmern voyait dans le nationalisme l'une des sources du bellicisme international, au même titre que les relations interraciales entre Blancs et non-Blancs et les relations économiques entre riches et pauvres (Zimmern 1934, 108). Selon lui, le nationalisme politique constituait une erreur française qui aurait fait coïncider indûment, lors de la Révolution de 1789, la liberté politique, c'est-à-dire les institutions libres de l'État, avec la conscience nationale, laquelle relèverait davantage d'une psychologie sociale (Zimmern 1929, 88). En raison de cette association de concepts étrangers l'un à l'autre, on aurait conclu à la coextension des limites de l'État à celles de la nation. L'idée générale derrière l'argument est bien connue, puisqu'il reproduit à peu de chose près la critique que Lord Acton formulait à l'endroit de J. S. Mill22. Selon Acton, non seulement les concepts de liberté et de nationalité ne coïncident pas, mais leur visée est souvent antithétique ${ }^{23}$. Dans ce contexte, le principe de nationalité doit être subordonné à celui de la liberté. Ainsi, « la nationalité [est] un élément essentiel mais non suprême dans la détermination des formes de l'État» (1907).

La solution que propose Zimmern à l'incommensurabilité de la liberté et de la nationalité diffère de celle d'Acton, d'abord parce que le principe de nationalité lui apparaît comme impropre au cadre politique. D'un point de vue théorique, il est contraire à l'idée de justice égale entre citoyens, puisque les résidents d'un territoire national appartenant à une nationalité autre que la dominante sont ipso facto réduits à une position d'infériorité civique. On reconnaît ici le moralisme politique de Zimmern, qui croyait fermement en un progrès moral en politique (Rich 2003, 89). D'un point de vue pratique, le principe de nationalité est contraire à la réalité effective des États nationaux, qui n'ont rien d'homogène, déstabilisant ainsi toute correspondance entre les institutions libres et les institutions nationales (Zimmern 1934, 171-3). De tels arguments sont aujourd'hui monnaie courante, et ont été repris par maints critiques du nationalisme, trop nombreux pour être ici nommés. Ce que propose Zimmern, cependant, ce n'est pas une subordination du principe politique de nationalité à celui de la liberté, comme le prônait Acton, mais la dépolitisation pure et simple de la nationalité et donc son exclusion hors de la sphère du politique (ibid., 186).

Or, force est de convenir qu'une nation ainsi dépolitisée se réduit à une communauté culturelle. Si, d'un côté, le politique repose sur un système de 
droit, un gouvernement et des institutions, la nationalité, elle, est selon Zimmern de nature culturelle. Les deux concepts occupent donc des espaces ontologiques distincts : « La question de la nationalité soulève un problème spirituel ou, si l'on veut, un problème culturel. [...] Il est tout à fait possible pour un groupe d'être à la fois politiquement autonome et culturellement immature, voire dépendant » (ibid., 175-6). Ce serait notamment le cas de la Nouvelle-Zélande qui, à l'époque, possédait en tant que dominion ses propres institutions gouvernementales, mais dont la culture - blanche, s'entend - dérivait de l'Angleterre. Inversement, il croyait qu' " il était possible pour une communauté sous le drapeau britannique d'être politiquement dépendante, mais culturellement indépendante » (ibid.), à la façon de l'Inde qui, à l'époque, était gouvernée par Londres mais possédait une riche culture qui lui était propre. C'est sur cette distinction fondamentale entre le politique et le national que Zimmern critique le rapport Durham, rédigé en 1839 à la suite des Rébellions de 1837, et que d'aucuns présentent comme un exemple d'arrogance ethnocentrique des Anglais. Selon lui, Durham aurait très bien saisi la dimension politique du problème en proposant la réforme des institutions dans le sens du gouvernement responsable. En revanche, il aurait complètement méconnu le problème de nationalité en cherchant à effacer la culture canadienne-française au profit de la culture anglaise. Il aurait confondu les dimensions politique et culturelle en cherchant à résoudre un problème strictement politique. Dans ce contexte, au lieu d'invoquer la supériorité culturelle de l'anglicité, qui émanait selon lui d'une « philosophie réactionnaire » (ibid., 178) ${ }^{24}$, il suffisait d'appliquer les principes politiques et légaux de la britannicité.

En somme, la dialectique de l'unité et de la diversité correspond chez Zimmern au rapport entre un unique État politique (comprenant ses lois, son gouvernement, ses institutions) et la pluralité des communautés culturelles en son sein. Ce que permettrait le Commonwealth britannique, c'est précisément l'harmonie de ces deux plans, ce qui n'est possible qu'en reconnaissant à chacun pour ainsi dire sa propre sphère ontologique et sa propre sphère d'action. Les problèmes émergeraient de la confusion des deux plans: « Au lieu d'associer et de confondre gouvernement et nationalité, [le Commonwealth britannique] a plutôt reconnu que l'art de gouverner consiste à subsumer différents types de gens, différentes nations, différents groupes, différentes religions, différentes cultures sous une seule loi, sous ce que nous appelons la pax britannica, sous un système international -j'insiste sur 1' "inter" - de justice » (Zimmern 1934, 174).

Ce passage et l'ensemble de la pensée de Zimmern peuvent être compris simplement en termes d' « ordre mondial », comme le font les quelques commentateurs de son œuvre ( $c f$. Miller 1979-80; Rich 2003). Mais la suite du texte est révélatrice quant aux contextes d'application de la dialectique de l'unité politique et de la diversité culturelle. Selon Zimmern, évoquant le droit à l'existence des nationalités au sein d'un État, « ce fut selon ce 
principe que le Canada français et le Canada britannique ont été, en 1867, unis dans une seule confédération » (Zimmern 1934, 174). La dialectique de l'unité politique et de la diversité nationale s'applique donc au dominion canadien, pour autant qu' « un dominion est une entité politique» (ibid., 175) permettant la subsomption de la diversité des nationalités, comprises somme toute chez Zimmern comme diversité des cultures. Zimmern se représentait ni plus ni moins un Canada multiculturel, dont le modèle est dû à l'union de l'Angleterre, de l'Écosse, du pays de Galles et de l'Ulster sous un seul gouvernement de la Grande-Bretagne. Ce modèle sera repris, comme le démontreront les prochaines sections, par les défenseurs de la britannicité au Canada. En outre, il est révélateur selon Zimmern que trois des cinq dominions aient été bilingues à l'époque (soit le Canada, l'Afrique du Sud et l'Irlande). Ainsi, l'union des nationalités ou des cultures sous un ordre politique ne correspond pas uniquement, chez Zimmern, à une vision internationaliste, mais à la forme accomplie d'un arrangement intra-étatique. En ce sens, le multiculturalisme est, si l'on veut, un internationalisme à petite échelle. Voilà ce que représente aussi à notre avis la britannicité du Troisième Empire.

Ce qu'il importe de mettre en relief dans le multiculturalisme intra-étatique de Zimmern, qu'il croit déjà reconnaître dans la constitution même du Canada, c'est une théorie « forte » de la coexistence des cultures. En d'autres termes, il ne s'agit pas simplement de tolérer la diversité culturelle. Le concept de tolérance est une notion trop passive. Il faut plutôt chercher activement à s'engager envers l'autre: « La tolérance n'est pas suffisante. Davantage est requis, à savoir la compréhension et l'effort d'accéder à une relation d'égalité » (ibid., 181). Sur la base de l'égale dignité des cultures, il faut en outre s'assurer que les communautés culturelles ne soient plus perçues par les autres ou par elles-mêmes comme inférieures ou « coloniales », mais qu'elles parviennent à un respect de soi: « le principe de nationalité [...] ne connaît que des communautés égales et respectueuses de soi-même » (ibid., 184). La diversité est ainsi perçue comme une richesse au bénéfice de l'humanité entière, permettant même une résolution plus aisée des nombreux problèmes politiques (ibid., 185-6).

À l'époque où Zimmern écrivait, il y a près d'un siècle, les intellectuels britanniques souscrivaient cependant toujours à l'idée d'une hiérarchie des civilisations, à l'instar d'Acton et de J. S. Mill, par exemple. Lorsque Zimmern invoque l'égalité des cultures, il entend donc l'égalité entre elles des « cultures avancées », excluant explicitement, par exemple, les habitants des îles du Pacifique ( $(\mathrm{bid}$.). Cependant, il ne faut pas y lire une quelconque hiérarchisation raciale, puisque l'Inde ou la Chine sont considérées comme de telles cultures avancées ${ }^{25}$. Il faudra attendre la fin de la Seconde Guerre mondiale pour que cette idée soit complètement discréditée. Mais déjà, durant la période d'entre-deux-guerres, il est permis d'affirmer que l'idée de multiculturalisme est d'ores et déjà établie comme norme occupant un espace légitime dans le discours public, et ce, en dépit des conditions auxquelles elle est encore assujettie. 
Évidemment, l'idée de multiculturalisme n'occupe pas à elle seule tout l'espace normatif du Troisième Empire. Elle est plutôt engagée, à cette époque, dans une espèce de compétition des normes, pour reprendre une expression du philosophe allemand Jürgen Habermas. C'est la raison pour laquelle Zimmern doit du même souffle polémiquer contre les anglocentristes de son époque. Certes, il fut un temps, désormais révolu selon lui, où la britannicité avait un sens ethnique. Toutefois, le terme « britannique » s'est à son avis dépouillé de ce caractère au Troisième Empire. «Aujourd'hui, [le terme "britannique"] n'a pas la moindre signification nationale. Si l'on vous disait aujourd'hui d'une personne qu'elle est britannique, aucune information ne serait communiquée sur sa langue ou sa culture. La britannicité a perdu sa teinte nationale pour devenir politiquement significative » (ibid., 180). Évidemment, la formulation descriptive de cette citation ne devrait pas occulter son sens normatif: selon Zimmern, le terme « britannique » devrait être dépourvu d'un sens national ou ethnique.

Sa critique d'une britannicité anglocentrique est virulente. Compte tenu de l'intense germanophobie ${ }^{26}$ des années où Zimmern a écrit The Third British Empire, en particulier dans le contexte de la montée du national-socialisme, on comprendra à quel point la comparaison qu'il établit entre anglocentrisme et idéologie allemande était caustique :

On ne peut trop insister sur ceci que toutes ces doctrines [anglocentriques] sont de pâles reflets de la doctrine allemande de la Kultur, tant réprouvée durant la guerre. Selon cette optique, il n'y aurait qu'une seule forme de culture toute-puissante et toute-bienveillante à laquelle les autres nations auraient intérêt à s'assimiler. Cette doctrine était en vogue en Allemagne et elle a causé énormément de tort. L'Empire britannique connaîtrait de sombres jours si nous l'adoptions, et ce, forcément, à une échelle bien plus grande. Ici encore, $[\ldots]$ nous sommes confrontés à une idée allemande que nous risquons d'appliquer sans en comprendre la véritable nature. (Zimmern 1934, 182; cf. aussi 1929, 92)

La théorie de la supériorité raciale serait du même acabit, et ne séduirait que l'homme blanc de basse condition (ibid., 112). Si l'idée de britannicité est normativement supérieure à celle ethnique d'anglicité, c'est précisément parce qu'elle est une notion politique et inclusive qui, de ce fait, est mieux à même de permettre une coexistence harmonieuse de la différence, source historique de conflits. Zimmern qualifie de « dangereuse » la conception ethnique de la britannicité, en particulier dans le contexte du Canada et de l'Afrique du Sud:

Les nouvelles communautés d'outre-mer incluent des populations considérables qui ne tirent pas leur origine de la Grande-Bretagne. Les Néerlandais, les Allemands et les huguenots en Afrique du Sud, les Canadiens français, les Slaves et autres immigrants de l'Europe 
continentale au Canada, sont près de constituer une majorité parmi les colonisateurs de ces deux dominions. Il y a un réel danger [...] qu'une trop grande insistance sur les liens ethniques, loin d'unir le Commonwealth, ne devienne au contraire la source de division à l'intérieur des dominions eux-mêmes. (Ibid., 97-98)

On peut donc reconnaitre en Zimmern l'une des inspirations intellectuelles du multiculturalisme canadien. Une telle idée serait cependant plus probante si on pouvait démontrer que la pensée de Zimmern a eu un écho avéré au Canada. Voilà ce que les deux sections suivantes se proposent d'accomplir.

\section{Le multiculturalisme du représentant de la Couronne britannique au Canada : John Buchan}

L'idée de britannicité-en-tant-que-multiculturalisme a été plus qu'une norme défendue par une classe intellectuelle anglaise durant la période d'entre-deux-guerres. On peut affirmer qu'elle a aussi représenté, en un sens, une norme sanctionnée par l'État canadien de cette époque, notamment en la personne de son gouverneur général, représentant de la Couronne britannique et chef d'État du Canada, à savoir John Buchan dit Lord Tweedsmuir ${ }^{27}$. C'est ce que confirment les discours de ce dernier ${ }^{28}$, lesquels auront permis que l'idée de multiculturalisme se déploie pour ainsi dire de façon endogène dans l'espace public canadien des années 1930. Ce déploiement aura été considérable, d'une part, en conséquence du prestige et de la dignité alors associés à la fonction de gouverneur général, mais aussi, d'autre part, en raison de la large diffusion médiatique dont ont fait l'objet ces discours dans les deux langues officielles. En effet, plusieurs de ceux-ci ont été radiodiffusés à travers le Canada, publiés et réédités dans de nombreux journaux du pays, ou ont paru dans un recueil de texte (Buchan 1940). Ce n'est pas à dire que le multiculturalisme ait été à l'époque une idée dominante ni qu'elle ait représenté une politique officielle du gouvernement canadien. Au contraire, le multiculturalisme de Buchan a été, semble-t-il, plutôt controversé, particulièrement auprès des Anglo-canadiens (Henshaw 2007, 201). En outre, il n'aurait pas été dicté par le gouvernement de Mackenzie King, bien que rien ne permette d'inférer qu'il s'y serait opposé (ibid., 194). Quoi qu'il en soit, l'idée de multiculturalisme s'établit désormais solidement dans le paysage normatif du débat public canadien (ibid., 203-205).

Ce lien étroit entre l'Empire britannique et le multiculturalisme canadien via John Buchan a déjà été mis en lumière par l'historien Peter Henshaw dans « John Buchan and the British Imperial Origins of Canadian Multiculturalism » (2007), un texte solidement étayé par une recherche minutieuse. Nos propos, bien que compatibles, ne se recoupent cependant pas entièrement. En effet, nous cherchons tous deux à démontrer l'origine britannico-impériale du multiculturalisme canadien, mais au lieu de circonscrire cette tâche en la personne de Buchan, nous nous sommes proposé de l'inscrire dans le contexte plus large 
du Commonwealth britannique et du sens de la britannicité durant cette période. En conséquence, Buchan apparaît ici comme une illustration canadienne de la façon dont l'idée de britannicité comportait une teneur multiculturaliste durant la période du Commonwealth britannique. C'est ce contexte qui a à notre avis rendu possible le déploiement des idées de Buchan sur le multiculturalisme.

En tant que représentant de la Couronne britannique au Canada, Buchan avait assurément un sens aigu de la dignité et des vertus de l'Empire. Sa description de l'Empire britannique fait écho à celle de Zimmern et à ses nombreux apologistes. Fait à noter, Buchan et Zimmern se connaissaient fort probablement et partageaient une vision similaire de l'impérialisme britannique. Tous deux faisaient partie de ce que Carroll Quigley a appelé le « Milner Group » (1981, ix $)^{29}$, un groupe d'oxfordiens ayant gravité autour d'Alfred Milner, administrateur colonial de l'Afrique du Sud au début du 20 siècle et ministre de la guerre dans le cabinet de David Lloyd George lors de la Première Guerre mondiale. Milner est connu pour avoir été un fervent impérialiste, champion de l'idée d'une fédération impériale. Selon Quigley, les membres du Milner Group, aussi connu en tant que « Milner's Kindergarten » ou « Round Table Group », parmi d'autres désignations, se connaissaient « intimement » (ibid.). Les membres de ce groupe ont par ailleurs joué un rôle important dans le cabinet de David Lloyd George lors de la Première Guerre mondiale. Plus spécifiquement, Alfred Zimmern a été une figure de proue du Political Intelligence Department, une unité sous la tutelle du Department of Information dont John Buchan a été le directeur ( $c f$. Smith 1996, 120).

Tout comme Zimmern, Buchan met en valeur le jeu dialectique de l'unité et de la diversité caractéristique du Commonwealth britannique, l'unité étant assurée chez Buchan par la Couronne:

Qu'y a-t-il de commun entre le Canada, une démocratie d'hommes blancs, et, disons, les îles Fidji? Ou entre l'Inde, pratiquant depuis peu le self-government et la Grande-Bretagne, qui se gouverne soi-même depuis mille ans? Qu'y a-t-il de commun entre des peuples de toutes les souches et de toutes les races de la terre? Pourtant, ces différences existent dans une unité. Le Trône unit l'ensemble de l'Empire et assure une cohésion à de vastes territoires dont la destinée ultime est imprévisible. $(1940,99)$

De façon similaire à Zimmern, Buchan croit aussi que l'Empire britannique constitue une sorte de partenariat de peuples distincts dont la finalité est d'assurer une pax britannica, à la façon de la Société des Nations. Discourant sur l'Empire, il dira peu de temps avant son arrivée au Canada que « nous sommes la preuve vivante de la capacité des peuples à vivre ensemble dans l'unité et la paix. N'avons-nous pas fait de l'Empire notre propre Société des Nations? Ne nous sommes-nous pas assurés qu'il ne puisse jamais y avoir de guerre sur une très grande portion de la terre? » $(1940,13)$. La diversité au 
sein de l'Empire constitue en elle-même une vertu qu'il s'agit de célébrer. Ainsi, dès son arrivée au Canada, il affirmera que « ce qui fait la gloire de notre Empire, c'est qu'il embrasse en son sein diverses races et traditions. C'est dans cette diversité que repose sa puissance » (tiré de Henshaw 2007, 192; cité aussi dans Saunders 2009).

Le socle britannique de la pensée multiculturaliste de Buchan se reconnaît par ailleurs à la valeur paradigmatique de la « scoticité » au sein de la britannicité. Buchan est en effet un Écossais ayant fait carrière dans l'Empire britannique. Rares sont ses discours où est omise toute référence à son Écosse natale. Il va sans dire que Buchan nourrissait une forte identité écossaise, laquelle cohabitait avec une forte identité britannique - teintée, il faut le dire, d'un élitisme oxfordien. Selon lui, l'Écosse reproduit doublement la dialectique de la diversité et de l'unité: à l'interne, puisqu'elle est le fruit d'une union de divers peuples, mais particulièrement de deux groupes ayant souvent été aux armes l'un contre l'autre, le Highland et le Lowland (Buchan 1940, 44; Henshaw 2007, 199); mais aussi dans son rapport à l'Angleterre (Buchan 1940, 44). La Grande-Bretagne est née de l'union de l'Écosse (et d'autres nations) à l'Angleterre, ce qui fera dire à Buchan que « nous les Écossais avons toujours été des champions de l'unité » (ibid.). Dans un élan quasi mystique, il semble attribuer à la « race » écossaise un pouvoir d'unification dont l'action ne devrait pas se limiter à la Grande-Bretagne et à l'Empire britannique (ibid., 32), mais devrait contribuer à l'idéal d'une " union spirituelle des nations du monde » (ibid., 45). Dans la mesure où, ayant « connu la folie de la division », l'Écosse aspire désormais à l'unité dans une structure politique englobante, elle devrait servir de modèle: " faisons de notre tradition écossaise une inspiration et un exemple » (ibid.). Finalement, l'enchâssement de la scoticité dans la britannicité constitue la preuve de la capacité de cette dernière à intégrer la diversité culturelle. C'est cette réalité qu'il s'agit d'adapter au contexte canadien.

C'est donc à travers le prisme de l'Écosse « britannique », distincte mais unie, que Buchan considère le Canada. En ce sens, « le Canada est simplement, en un sens, l'Écosse à plus grande échelle » (ibid., 39). Il réitère en maints endroits que c'est en tant qu'Écossais qu'il se doit d'insister sur le caractère culturellement pluriel de la nation canadienne. Ainsi, au Congrès de la langue française, il dira en 1937: « Tout homme sage, tout Écossais en particulier, croit certainement que le mélange des races fortifie une nation » (ibid., 243).

Par conséquent, Henshaw a raison de voir dans la pensée de Buchan une anticipation du multiculturalisme canadien. Les discours de Buchan sont très éloquents à cet effet. Y sont discernables différentes dimensions du multiculturalisme canadien. Tout d'abord, dans toute sa généralité, on retrouve la dimension attitudinale d'ouverture envers l'autre, c'est-à-dire envers les individus d'origines, de religions et de cultures différentes. À la façon de l'humanisme nationaliste de Herder et de Charles Taylor (1992) à sa suite, il 
s'agit de reconnaître une certaine valeur intrinsèque à toute culture étrangère. Une telle perspective est certes présente chez Buchan, comme en témoigne ce passage: "Il nous revient d'étudier l'esprit des peuples étrangers et d'y chercher le noyau de vérité, l'élément de valeur qui s'y trouve nécessairement » (Buchan 1940, 83). En ce sens, la diversité est valorisée en et pour elle-même.

Or, si toutes les cultures ont une égale dignité, elles peuvent, voire doivent toutes être célébrées également. L'anglo-conformité, dans la mesure où elle transgresse ce principe, est ainsi invalidée ipso facto. C'est la raison pour laquelle Buchan insiste souvent sur le devoir de mémoire qui incombe à toutes les communautés culturelles du Canada et sur l'importance de revendiquer ses racines, d'où l'intérêt de la dimension historico-identitaire. Ce message est un leitmotiv des discours de Buchan, qu'il a sans cesse adapté selon le groupe ethnique auquel il s'adressait. À la communauté islandaise de Gimli, qu'il félicite comme " de bons Canadiens dans le plein sens du terme », il dira: « je me réjouis à l'idée que vous n'ayez jamais oublié les traditions de votre mère patrie » (ibid., 27). Aux Écossais de Winnipeg réunis à l'occasion de la Saint-André, il dira: « Nous avons une tradition à préserver, une pleine tradition. Voilà notre responsabilité première. Nous avons aussi un devoir à l'égard de la patrie de ces traditions - notre lieu de naissance ou celui de nos aïeux ». Il s'agirait alors de « maintenir un vif intérêt non seulement pour notre race, mais pour la mère patrie » (ibid., 42-43; cf. aussi ibid., 32 et Gibbon 1938, 307). À la communauté ukrainienne, qu'il reconnaît aussi comme de " bons Canadiens », il adressera une phrase lapidaire: "Vous serez tous de meilleurs Canadiens en étant aussi de bons Ukrainiens » (Gibbon 1938, 307). Et de même pour la communauté francophone du Québec : "Vous avez conservé votre langue, vos droits, votre religion et votre culture si riche d'histoire, d'un si grand prix pour le Canada tout entier » (Buchan 1940, 243). On est donc bien loin de l'anglocentrisme attribué généralement aux Britanniques de cette époque.

Cette dimension historico-identitaire a des conséquences pour la dimension politico-identitaire ou la dimension nationale du multiculturalisme canadien. En effet, elle entraîne d'une part, au niveau de l'individu, la nécessité d'admettre une multiplicité d'allégeances éventuelles dans la formation d'une identité nationale individuelle. Tout être humain, pour libre qu'il soit, s'enracine toujours dans des formes de vie concrètes auxquelles il ne peut échapper : « Il est assujetti à des loyautés à la famille, à la race et à la terre natale, à la paroisse, à la province et à la nation; de même, il est pourvu d'une multitude de traditions qui lui sont chères » (ibid., 158). Il s'agit alors de concilier ces loyautés ou ces « patriotismes » étroits ou locaux en un patriotisme plus large, ce pour quoi les Écossais auraient, selon Buchan, un talent particulier. Dans les mots de ce dernier : " on ne peut jamais avoir trop de loyautés. Ainsi, j'aimerais trouver parmi les citoyens canadiens un amour fort et durable pour la région à laquelle ils appartiennent, mais en même temps un intérêt fort et durable pour le dominion dont ils font partie, c'est-à-dire l'ensemble de la 
nation canadienne » (ibid., 32). D'autre part, l'attachement aux racines diverses entraîne, au niveau de la collectivité, la valorisation de la diversité comme source d'une identité nationale commune. L'idée de diversité se doit alors de s'insérer dans l'identité canadienne. Une tâche nationale d'intégration est ainsi définie : « [Le Canada] doit chercher à unir, dans un patriotisme commun, des citoyens de plusieurs races » (ibid., 189).

Sur ces points touchant à la valorisation du multiculturalisme comme projet national chez Buchan, et donc sur l'essentiel, Henshaw et nous sommes du même avis. Cependant, là où Henshaw adopte une attitude apologétique quant à l'inclusivité du projet multiculturel tel que l'entendait Buchan, il convient à notre avis d'être un peu plus critique. Henshaw concède que Buchan avait une certaine prédilection pour les cultures nordiques et pour la religion chrétienne, mais que, somme toute, une telle préférence vénielle ne minait pas le fondement normatif de son multiculturalisme (Henshaw 2007, 202). Certes, Buchan aurait eu un passé raciste, ce dont ses romans témoignent $(\text { ibid., } 191)^{30}$. Mais il aurait progressivement abandonné ses préjugés anglocentriques, au point où, en tant que gouverneur général du Canada, il aurait pleinement épousé un humanisme multiculturel. Buchan lui-même semble faire écho à cette interprétation génétique lorsqu'il parle au passé de « ce qui fut un jour nos grands défauts, à savoir le chauvinisme et l'orgueil racial. Or, nous en savons davantage sur les autres peuples, et ce savoir a engendré une sympathie non seulement de la tête, mais aussi du cœur. Un tel patriotisme de l'humanité [...] n'est en aucun cas incompatible avec le patriotisme national [...]» (Buchan 1940, 126).

Il faut convenir que Buchan a souvent défendu l'idée d'un tel patriotisme de l'humanité dans ses discours. Il exalte volontiers une « unité spirituelle des nations du monde » (ibid., 45) ou une " puissante sympathie à l'échelle humaine » (ibid., 159). Ce qu'il estime particulièrement dans le scoutisme, par exemple, c'est l'adhésion « de centaines de milliers de membres de toutes les races et nations » (ibid., 143). De telles affirmations sont monnaie courante dans les discours de Buchan. Néanmoins, quelques passages clairement contraires à l'esprit de l'harmonie multiculturelle des peuples ne peuvent être ignorés dans ce contexte. C'est le cas notamment d'une opinion exprimée dans le tout premier discours de Buchan en sol canadien. Après une description de ce qu'il considère comme l'essence de la culture occidentale, il dira: « Il existe d'autres cultures dans le monde, chacune avec sa propre valeur pour son propre peuple. Je ne les critique pas, sauf pour dire qu'elles ne sont pas les nôtres et qu'elles ne se mélangent pas bien avec la nôtre. Il y a aujourd'hui beaucoup d'anarchie dans notre art et notre littérature causée par l'intrusion d'éléments étrangers - slaves, mongols, négroïde - dans une sphère où ils n'ont aucune place » (ibid., 22). Ces paroles sont plutôt surprenantes pour un apôtre du multiculturalisme et elles vont bien au-delà de la forme bénigne de chauvinisme anglocentrique ou occidental, présente çà et là dans ses discours (p. ex. ibid., 60, 65). On sait que, plus tard, il reviendra sur son appréciation 
des Slaves dans son discours de Fraserwood, où il affirmera, à l'encontre de l'esprit de son discours inaugural au Canada, que « l'élément ukrainien est une contribution très valable à notre nouveau Canada » (Gibbon 1938, 307). Mais on ne note nulle part dans ses discours un tel revirement quant à son appréciation des Asiatiques et des Noirs. En fait, le mutisme général de Buchan sur la place qui revient aux Noirs et aux Asiatiques est en lui-même suspect. Il semble donc que son multiculturalisme ne soit pas tout à fait à la hauteur des normes qu'il défend.

Par conséquent, s'il faut interpréter la profondeur du projet multiculturel de Buchan en termes génétiques, force est de conclure que son évolution était loin d'être terminée lors de son mandat à la tête de l'État canadien. Nous croyons que Buchan défendait un hiérarchisme culturel qui ne fut pas sans effet sur l'idée qu'il se faisait personnellement du multiculturalisme, mais qui ne se traduit pas non plus par un multiculturalisme de l'homme blanc. À la façon des penseurs victoriens de la diversité, tel Lord Acton (1907), il croyait à l'existence d'une certaine hiérarchie des civilisations où, généralement, les Blancs (nordiques avant tout) tenaient le haut du pavé, mais où les civilisations orientales pouvaient théoriquement aussi avoir une place. En ce sens, Buchan semble avoir reçu favorablement l'idée d'une Inde se gouvernant soi-même $(1935,283-286 ; 1940,99)$. Mais il n'y a aucun doute dans son esprit quant à une hiérarchie des civilisations. Comme il le note en 1938 dans un discours au Collège Bishop à Lennoxville, ceux occupant les niveaux les plus bas « dans l'échelle de développement» sont selon lui " les peuples de couleur (dark-skinned) en Asie, en Afrique et dans les îles des gouvernements de la mer » (ibid., 99-100). Même si on cherchait à défendre Buchan en distinguant une inégalité culturelle de fait - et donc contingente - d'une égale dignité des cultures, les propos semblent déplacés, surtout selon les normes d'aujourd'hui.

Henshaw concède la disposition hiérarchiste de Buchan lorsqu'il affirme qu' « il est aussi vrai que Buchan croyait dans une hiérarchie de peuples ou de nation, une hiérarchie où la race britannique et d'autres races "nordiques" occupaient le sommet » $(2007,191)$. Mais selon l'auteur, une telle croyance ne s'opposait pas au projet multiculturaliste d'une nation canadienne inclusive et respectueuse des origines multiples de ses citoyens. Or, dès que l'on exprime, publiquement de surcroît, l'infériorité - provisoire ou non - d'un peuple, il semble évident que l'on contrevient du même coup au devoir de respect à son égard, et ce, en dépit de toute « bienveillance » que l'on pourrait lui témoigner par ailleurs. Dans ce contexte, il est difficile d'affirmer que Buchan défend « une approche à la culture et l'identité canadiennes qui accommoderait sans peine des individus de toutes les parties du monde » (ibid., 203) sans y apporter un bémol.

Cela dit, l'interprétation que propose Henshaw du multiculturalisme des années 1930 à la lumière de Buchan est fort intéressante : il rejette le modèle 
linéaire d'une succession d'idéologies menant de l'assimilationnisme (dans la forme d'un anglo-conformisme ou du melting pot) au multiculturalisme. Selon lui, il n'y avait pas à choisir entre ces idéologies, ce que Buchan aurait lui-même évité de faire. En effet, selon Henshaw, Buchan aurait adopté à la fois un anglo-conformisme bénin en faisant des cultures britanniques un modèle à suivre, l'idéologie du melting pot en prônant un nationalisme proprement canadien, et l'idéologie multiculturaliste en exhortant les individus à préserver leur culture particulière (Henshaw 2007, 206). Néanmoins, nous croyons que cette thèse ne peut être défendue qu'en entretenant un certain vague dans la signification des termes. D'abord, l'anglo-conformisme est ici dilué et détourné de son sens fort et assimilationniste, dans la mesure où il prend une valeur vaguement paradigmatique au lieu de servir de critère d'assimilation. Il en va de même pour l'idée de melting pot lorsqu'il se fonde sur le respect de la diversité culturelle au lieu d'assumer son sens foncièrement assimilationniste. En ce sens, on demeure un défenseur du multiculturalisme même si l'on prône l'encadrement de la diversité par une unité intégrée. Will Kymlicka a bien montré que le multiculturalisme canadien inclut aussi un principe d'intégration, la pluralité culturelle assurant quant à elle un critère d'équité (fairness) à l'intégration (2003, 65-). Ainsi, Henshaw confond assimilation, melting pot et multiculturalisme pour ne retenir finalement que l'idée d'un multiculturalisme empreint d'un ethnocentrisme bienveillant.

Néanmoins, nous croyons que Henshaw a raison de vouloir briser le modèle trop linéaire d'une succession d'idéologies discrètes. Le nationalisme multiculturaliste canadien des années 1960 et 1970 ne s'est pas simplement imposé en opposition à la britannicité du $19^{\mathrm{e}}$ et de la première moitié du $20^{\mathrm{e}}$ siècle. Nous croyons nous aussi qu' « il est ironique qu'une grande part du vide créé par la renonciation aux symboles britanniques au Canada ait été comblée par une idéologie ayant de profondes racines britannico-impériales, une idéologie conçue et défendue initialement et éminemment en vue de maintenir l'Empire » (Henshaw 2007, 205). Mais si cette affirmation nous paraît valide, ce n'est pas parce que les idéologies assimilationnistes (l'angloconformisme et le melting pot) et le multiculturalisme pouvaient cohabiter de façon cohérente dans une même pensée, mais parce que l'idée même de britannicité incluait alors en elle le principe multiculturaliste. En même temps, cependant, l'universalisation de ce principe n'avait pas encore été accomplie en raison d'un certain hiérarchisme racial toujours présent en arrière-plan, bien qu'aplani depuis la fin de la Première Guerre mondiale.

Enfin, il est important à notre avis, pour éviter toute confusion, d'établir une distinction nette entre faits et normes. Nous croyons que le multiculturalisme normatif a acquis ses lettres de noblesse durant la période du Commonwealth britannique, comme le démontre la place qu'il occupe dans la pensée d'intellectuels tels que Zimmern et Buchan, et qu'il a ainsi gagné en importance dans le discours public canadien - ce que confirme Henshaw (2007, 
203-205). Cependant, il ne s'ensuit pas qu'il ait été communément pratiqué par la population ou même adopté comme politique publique au Canada. Il fallut attendre les années 1970 pour que le multiculturalisme devienne politique publique au Canada. Quant à son intégration dans la pratique populaire, il s'agit là d'un projet en chantier, toujours à refaire.

\section{Britannicité et minorité canadienne-française: l'exemple d'Édouard Montpetit}

Zimmern reconnait dans The Third British Empire que l'Empire a d'abord été fondé sur la force et la coercition. Mais selon lui, la force à elle seule est insuffisante pour assurer l'union d'un Empire : « Nous avons conquis le Canada français, l'Inde et de grandes portions de l'Afrique, directement ou indirectement. Cependant, bien que nous y ayons acquis nos positions par la force, nous ne les maintenons pas par la force » (Zimmern 1934, 101). À la force a succédé ce qu'il appelle un « assentiment passif», rapidement acquis selon lui au Canada français grâce à une politique de prudence, de tolérance et d'apaisement, mais aussi grâce au prestige dont jouissait alors l'Empire.

Il serait donc faux de comprendre le Canada français, comme le fait Carl Berger (1970, p. ex. 145, 232) parmi tant d'autres, uniquement comme vecteur antibritannique à partir de la fin du $19^{\mathrm{e}}$ siècle. Tel que le notent Phillip Buckner et R. Douglas Francis (2006, 3), bien que les Canadiens français n'eurent jamais été aussi enthousiastes à l'idée de l'impérialisme britannique que les Canadiens anglais, l'élite canadienne-française n'y a jamais été complètement opposée, tout au contraire ( $c f$. Thompson 2008, 91). Il convient en fait de préciser davantage cette pensée à l'aide d'un passage des écrits d'Henri Bourassa. John Darwin, dans le quatrième tome du monumental Oxford History of the British Empire, fait allusion à la caractérisation par Bourassa, un «nationaliste canadien-français ", du système impérial britannique comme " monstrueux empire » (Darwin, 1999, 70). Or, on peut retracer ces paroles dans une lettre écrite en anglais par Bourassa en 1916, dans laquelle on lit ce qui suit:

One point I did not develop in our conversation is the motive of my desire for the disruption of the British Empire. It is not because it is British, but because it is Imperial. All Empires are hateful. They stand in the way of human liberty, and true progress, intellectual and moral. They serve nothing but brutal instincts and material objects. All that is good in British ideals, and there is much of it, would be better served by the free action of several independent British communities than by the common action of a monstrous Empire, built up by force and robbery; and kept together for no other purpose than allowing one race and one nation to dominate one-fifth of the human race. British nations have to choose between British ideals and British domination. I stand for ideals against domination. I may be hanged for it, in the name of British liberty, but that does not matter. (Bourassa 2009) 
Bourassa, semble-t-il, aurait voulu prendre à partie la teneur proprement impériale de l'Empire britannique, mais pas sa nature britannique. L'impérialisme est par essence hégémonique et coercitif et, de ce fait, contraire à l'idéal de la liberté. La britannicité, elle, renvoie à des idéaux nobles, tels que la liberté et l'inclusion. Elle renvoie, comme Bourassa le nota dans un écrit antérieur, à des institutions que les Canadiens français ont librement contribué à façonner (Bourassa 1903, 9-10)31. Coates a donc raison de prétendre que le rapport des Canadiens français à l'Empire britannique est ambigu, et l'a toujours été. Selon lui, on y a loué, au cours des siècles, les « libertés britanniques » tout en cherchant à maintenir le plus haut degré d'autonomie (Coates 2008). Selon Sylvie Lacombe, les élites politiques du Canada français considéraient naturellement, dès le $19^{\mathrm{e}}$ siècle, leur peuple comme des britanniques de langue française, jouissant des mêmes droits et privilèges que tous les autres sujets britanniques (Lacombe 2006).

Dans la suite du texte, nous aimerions mettre en évidence la façon dont l'idée de britannicité associée à une pensée du multiculturalisme est aussi présente au Canada français. Notre attention portera sur une figure modérée du nationalisme canadien-français de l'entre-deux-guerres. Il s'agit d'Édouard Montpetit (1881-1954), avocat et économiste, intellectuel public et fondateur de l'École des sciences sociales, économiques et politiques de l'Université de Montréal. Le nationalisme de Montpetit est tout entier ancré dans le Zeitgeist du Québec des années 1920 et 1930. Il est fortement marqué par l'empreinte de la foi catholique et par un sentiment d'urgence quant à la défense de la langue française. On sait par exemple qu'il collabore à la revue clérico-nationaliste L'Action française, dirigée pendant plusieurs années par le chanoine Lionel Groulx, et plus tard à la revue L'Action nationale, l'organe de la très nationaliste Ligue d'action nationale. Notons par ailleurs que durant la période d'entre-deux-guerres, le nationalisme canadien-français se préoccupait particulièrement de la formation d'une élite qui pourrait prendre les rênes de la destinée nationale. La survie de la nation dépendait de la formation d'experts en économie, en finance et dans les sciences sociales. Dans ce mouvement, on retrouve Athanase David, Victor Doré et Édouard Montpetit (Fournier 1982)32, parmi d'autres. Ce dernier affirmait dès 1917 que « la question nationale est d'abord une question économique [...] et c'est l'instruction qui nous assurera la conquête économique (Montpetit 1917, 315; tiré de Fournier 1982, 5n; $c f$. Montpetit 1939). La suite de son œuvre fera de ce souci un mantra (cf. en particulier Montpetit 1939, chap. I).

Cependant, si Montpetit était un nationaliste convaincu, défenseur indéfectible de la langue française et de la culture canadienne-française, il n'en était pas moins fédéraliste, ouvert au Canada anglais et aux États-Unis, et soucieux de contribuer à la vitalité de la fédération canadienne. Il croyait en la nécessité d'un rapprochement entre les Canadiens anglais et les Canadiens français $(1937,37)$ avec pour fin une unité nationale canadienne (cf. ibid., 49). Pour 
Montpetit, nul doute que les deux civilisations ne s'enrichissent mutuellement. Notons d'emblée que Montpetit défend explicitement un biculturalisme anglais et français plutôt qu'un multiculturalisme. Néanmoins, fait intéressant pour notre propos, il défend ce biculturalisme à l'aide de la pensée zimmernienne de la britannicité et de la valeur inhérente de la diversité en elle-même, ouvrant de ce fait - et sans doute malgré lui - la porte au multiculturalisme. En effet, si on peut justifier la cohabitation de deux cultures par l'idée de britannicité et de diversité, on peut le faire pour plusieurs. C'est la raison pour laquelle Montpetit nous intéressera particulièrement ici.

Un écrit retiendra particulièrement notre attention. Il s'agit de « Sommesnous en pays britannique? $»^{33}$, tiré de son ouvrage de 1937 intitulé $D^{\prime}$ azur à trois lys d'or, qui regroupe une série de conférences présentées à des auditoires anglophones. Une anecdote sert de point de départ à la réflexion entamée par Montpetit sur le sujet de la britannicité. Il raconte en effet l'histoire d'un touriste anglais égaré à Montréal durant les années 1930. Après avoir essuyé l'incompréhension de nombreux francophones à qui il s'était adressé, le touriste réussit finalement à se faire comprendre par un Écossais, collègue de Montpetit à l'Université de Montréal. Se sentant enfin en compagnie d'un compatriote, le touriste se permit de pester contre les Canadiens français : « Nous devrions les jeter tous à la mer! » Mais ce sont les paroles suivantes qui engagent sa réflexion: « Après tout, dira le touriste anglais, nous sommes en pays britannique » (Montpetit 2005, 120).

Or, selon Montpetit, l'idée même de britannicité n'a rien à voir avec un tel chauvinisme, tant s'en faut. À l'appui de sa thèse, il fait explicitement appel à la pensée d'Alfred Zimmern et, implicitement, à son ouvrage The Third Empire ${ }^{34}$. De façon conforme à ce dernier, Montpetit identifie deux caractéristiques principales de l'Empire britannique: la diversité et l'unité, qu'il comprend lui aussi dans un rapport dialectique. La véritable force de cohésion de l'Empire est selon Montpetit le respect de la diversité. Il évoque la cohabitation dans l'Empire de cultures diverses et insiste particulièrement, comme l'ont fait Zimmern et Buchan, sur la présence de cette diversité au sein même de la Grande-Bretagne, où coexistent les cultures galloise, écossaise, cockney et autres. Parmi les diverses cultures de l'Empire, il signale les cultures indiennes, marquées elles-mêmes par une complexe diversité, mais aussi les Afrikaners et les Québécois (2005, 122; 1937, 17). L'unité dans la diversité résulte selon Montpetit des libertés et de l'égalité accordées - à la suite de longues luttes, certes - à ces groupes nationaux. C'est ce qui lui permet d'affirmer que «l'unité est [...] un phénomène d'émancipation » (ibid., 123; 1937, 18-19). Cette « unité [des] traits mobiles » serait à la source de la puissance impériale britannique.

Dans ce bref écrit sur le sens de la britannicité, mérite avant tout notre attention la thèse selon laquelle l'Empire britannique, en tant que symbole de 
diversité culturelle uni par des principes de liberté et d'égalité, devrait être érigé en modèle normatif pour le Canada lui-même:

Le problème de l'unité canadienne serait résolu si nous appliquions dans son ampleur le principe de la puissance impériale. Le Commonwealth se dresse devant nous comme un exemple. Le Canada est un pays britannique en ce sens qu'il est un pays de diversité. Être un pays de civilisations différentes, de langues différentes, de cultures et de religions différentes, c'est comme on dit aujourd'hui, synchroniser l'Empire. (Montpetit 2005, 124; 1937, 20)

Cette idée n'a rien d'isolé dans son œuvre ${ }^{35}$. Ainsi, le Canada devrait être à petite échelle ce que l'Empire britannique est à grande échelle. Il n'est «britannique », dans son sens fort, que pour autant qu'il tende vers l'idéal de la diversité des cultures.

Pour Montpetit, cette formulation en termes normatifs de la canadienneté exprime du même coup un état carentiel, celui d'un Canada qui n'est pas à la hauteur des normes de la britannicité. Et si l'on garde à l'esprit que Montpetit s'adresse dans ce texte à un public anglophone, il est clair que le reproche d'un État chauvin n'épousant pas la diversité effective en son sein est formulé à l'encontre du Canada anglais. « Pourquoi, quand il s'agit du Canada et non plus de l'Empire, du Canada partie de l'Empire, repoussons-nous la diversité, source d'unité? Pourquoi être impérialiste dans l'Empire, et nationaliste - on sait de quel nationalisme étroit - au Canada? Pourquoi louer les libertés impériales si c'est pour en priver notre pays? » $(2005,124 ; 1937,19-20)$. Il s'agit là pour Montpetit d'une absurdité. Au fond, il fait grief aux Canadiens anglais de ne pas être assez britanniques. Si le Canada est, il doit être britannique, c'est-à-dire multiculturel au sens où l'on célèbre la diversité de cultures libres et égales.

Certes, lorsque Montpetit chante les louages de la diversité ethnique, il a spécifiquement en tête la cohabitation des cultures anglaise et française. $\mathrm{Sa}$ pensée réelle est révélée par ce passage: «Si des dominions divers sont un actif pour le Commonwealth, nos deux civilisations enrichissent notre pays de la même façon. C'est pourquoi nos deux "éléments" doivent garder et fortifier leurs qualités essentielles. [...] Les deux groupes ethniques, conscients de leur valeur humaine et forts de leur apport séculaire, collaboreraient à la grandeur commune [...]» $(2005,125 ; 1937,20-21)$. Dans l'idée qu'il se fait d'un Canada multiculturel, il ne semble pas envisager la participation des cultures autochtones, ukrainienne dans les Prairies, japonaise sur la côte Ouest. Il n'en demeure pas moins que l'idée normative, celle de la cohabitation d'une pluralité de cultures, de langues, de religions, de modes de vie, celle du rejet de "l'égoïsme de race » (ibid., 129; 1937, 26) est désormais établie avec force. L'être - c'est-à-dire le « fait »-d'une diversité plus extensive que ne l'envisageait Montpetit ne change rien au devoir-être de la norme ainsi reconnue. 
Enfin, notons que si la diversité est mise en valeur, comme on l'a vu, pour des raisons éthiques (et sans doute aussi esthétiques), elle l'est aussi, négativement, dans sa fonction de bouclier contre les forces homogénéisantes de la modernité ( $c f$. Montpetit 1937, 44-45), de l'américanisme en particulier (cf., Montpetit 2005, 127; 1937, 23-24). Cette position n'est pas sans rappeler la célèbre thèse que le philosophe canadien George Grant a défendue bien des années plus tard dans Lament for a Nation (1965; en français: 1987). Chez Grant, cependant, c'est le pessimisme qui l'emporte puisque, selon lui, la partie était d'ores et déjà perdue: la nation canadienne se résorbait graduellement devant l'avancée de l'américanisme (son libéralisme, son capitalisme, son scientisme). Dans la période de l'entre-deux-guerres, Montpetit présente la diversité britannique dans son opposition à l'homogénéisation américaine. Ainsi, en un sens, c'est la vitalité de la culture canadienne-française qui sert de rempart contre la disparition culturelle du Canada anglais devant la menace américaine:

Les groupes, maintenus jusqu'ici dans notre pays, avec leurs coutumes, leurs traditions, leurs façons dissemblables de penser, d'envisager la vie, voilà l'originalité qui noue notre résistance à la standardisation où sombrent les masses américaines. [...] À quand la fin du Canada anglo-saxon privé de notre vigueur? Car si l'on croit angliciser le Canadien français, on se trompe: libéré de ses attaches, délié de sa race, il s'américanisera sûrement. (2005, 127; 1937, 23-24)

Déjà à cette époque, on pressentait une distinction essentielle entre la diversité culturelle à l'américaine et à la canadienne. Car s'il n'y avait pas de doute pour Montpetit que les deux pays étaient nés de la diversité (il dira par exemple «le Canada, comme les États-Unis, a convié le monde sur son territoire», ibid., 122; 1937, 17), le propre du Canada se reconnaissait à sa capacité à la préserver. Pas étonnant, alors, que pour se démarquer du modèle américain du melting pot, la formule de la «mosaïque canadienne » soit née durant la période qui nous intéresse, celle qui a vu naître le Commonwealth britannique.

\section{Conclusion}

Le multiculturalisme canadien n'est donc pas le simple produit d'une histoire récente. Il est une façon de voir le monde ayant été préparée de longue date. Tel que démontré, on retrouve le germe de cette vision du monde dans le bagage normatif du Troisième Empire britannique. En ce sens, on pourrait dire que le multiculturalisme d'État qu'on connaît au Canada aujourd'hui est l'aboutissement de la britannicité-en-tant-que-multiculturalisme telle qu'elle s'articulait dans la période d'entre-deux-guerres, et apparaît comme un produit postcolonial. Certes, il n'est pas question ici d'argumenter une quelconque réalisation effective du projet multiculturel à cette époque, puisque l'anglocentrisme représentait sans nul doute le modèle dominant dans le rapport des cultures au Canada, bien que le premier semble l'avoir subséquemment emporté 
sur le second dans la compétition de normes qui les opposait. Cependant, toute prétention à une réalisation effective du multiculturalisme est d'autant plus saugrenue qu'aujourd'hui encore, on serait en peine d'affirmer que le multiculturalisme est chose faite. Les conflits entre cultures existent toujours au Canada comme ailleurs, mais l'idéal - c'est-à-dire la norme - de la cohabitation des cultures, désormais dominant, pousse le public canadien à chercher à y répondre avec vigueur. Voilà l'essentiel, à savoir la reconnaissance du multiculturalisme comme norme établie. Or, l'établissement du multiculturalisme comme norme a son origine selon nous dans la période du Troisième Empire britannique. En ce sens, une certaine britannicité s'est maintenue au Canada, lors même qu'on croyait s'en défaire.

\section{Notes}

1. Pour une telle analyse historique de l'origine du multiculturalisme canadien, $c f$. $\mathrm{p}$. e. Wayland (1997).

2. On songe en particulier à Mathieu Bock-Côté (2007 et 2008) chez les Québécois, à Bibby (1990), Stoffman (2002), Gwyn (1995) et Bissoondath (1995) au Canada anglais.

3. Son défenseur le plus éloquent est sans nul doute Will Kymlicka. Cf. 1998.

4. C'est le cas notamment du Parti conservateur du Canada, sans doute désireux d'acquérir un certain crédit pour le multiculturalisme, normalement associé au Parti libéral du Canada, auprès des communautés ethniques. Voir le discours du ministre de la Citoyenneté, de l'Immigration et du Multiculturalisme, Jason Kenney (2009).

5. D'autres auteurs ont préféré le contexte qu'offrait le Commonwealth moderne, après la Déclaration de Londres de 1949 qui, entre autres choses, éliminera l'épithète « britannique » du Commonwealth. W. L. Morton, par exemple, décrit le Commonwealth comme une " association libre qui, à son mieux, transcende les différences de race et de culture » et dont la « signification première » repose sur son « caractère multiracial» $(1972,56)$. Selon Morton, les Canadiens devraient reconnaître dans l'évolution du Commonwealth « une victoire de l'expérience canadienne » (ibid., 57). Toutes les traductions sont de nous.

6. Will Kymlicka a aussi recours à ce sens large dans certains de ses écrits. $C f$. par exemple 2005, 54 sqq. et 2006, 23 sqq., où il inclut les immigrants, les minorités nationales et les minorités autochtones dans son étude des politiques multiculturelles. Dans 2006, on y retrouve de plus les métèques (metics).

7. Le tournant du $20^{\mathrm{e}}$ siècle est particulièrement riche d'exemples faisant valoir le rôle de l'Empire britannique comme unité dans la diversité des cultures. $C f$. par exemple, Lacombe (2002), Cannadine (1997, p. 1972), et Wardhaugh (1997).

8. Le renouveau des nationalismes écossais, anglais et gallois serait ainsi le symptôme de cet effritement de la britannicité. $C f$. Colley (2003, 6-7).

9. Cependant, la relation entre l'Irlande et la Grande-Bretagne a toujours été complexe. Cf. p.e. Holmes (2001). 
10. La préface de l'ouvrage débute ainsi: « En 1866 et 1867, j’ai parcouru l'Angleterre tout autour du monde; j'étais partout dans des pays anglophones ou gouvernés par les Anglais » (ibid., ix). Cf. aussi du même auteur, Problems of Greater Britain, où il décrit ainsi son objectif: « réexaminer, après un intervalle de vingt ans, les terres où prévalent le gouvernement anglais ou la langue anglaise » (1890, viii).

11. Une telle assertion ne s'oppose pas au fait qu'il existait aussi, en Amérique du Nord britannique, des patriotismes provinciaux ou des tenants d'un nationalisme canadien, comme dans le mouvement Canada First. Sur ces questions, voir Taylor (1989). Selon Canny, les colons américains issus de l'Angleterre, de l'Écosse, du pays de Galles et de l'Irlande se considéraient comme britanniques dès le $17^{\mathrm{e}}$ siècle $(1998,24-25 ; 372-373)$.

12. Cf. Zimmern $(1934,181)$ : «Puis, graduellement, il [le terme "britannique"] s'est étendu pour inclure les Blancs d'outre-mer et il est encore souvent employé en ce sens aujourd'hui [les années 1930] - les peuples à peau blanche sous ledrapeau britannique ».

13. Notons que dans la Loi sur la citoyenneté canadienne de 1947, les citoyens canadiens demeuraient des « sujets britanniques ». Dans la Loi sur la citoyenneté canadienne de 1977, le terme « sujet britannique » a été remplacé par « citoyen du Commonwealth».

14. Pour une description plus nuancée de ce repli après la Seconde Guerre mondiale, en particulier dans son rapport aux nouvelles lois sur la citoyenneté et à l'européisation de la Grande Bretagne, $c f$. McIntyre (2009, 66-72).

15. Pour une discussion circonstanciée de la fin de l'Empire britannique, voir Darwin (2005).

16. L'ouvrage de H. Duncan Hall, The British Commonwealth of Nations, 1920, est le premier à paraître sur le sujet.

17. Pour une comparaison de ces trois auteurs entre eux, $c f$. Miller (1979-80).

18. Cf. Markwell (2004); McIntyre (2009, 31-37) pour une courte biographie; Rich (2003, 80-82) pour des données biographiques. Selon Rich (2003, 81), Einstein, Bartok et Bergson faisaient partie du comité présidé par Zimmern.

19. Dans ce contexte, Zimmern aime à dire que l'Empire britannique « a rassemblé un quart de l'humanité sous une seule loi » (104).

20. La suite de l'histoire postérieure à la publication de l'ouvrage de Zimmern, en particulier depuis la Seconde Guerre mondiale, rend obsolète la critique de Hancock selon laquelle l'hypothèse de la marche des communautés vers l'autodétermination était un leurre. (Hancock, 1937, 393n, 489; cité dans Miller, 1979-80, 160).

21. Dans un autre passage, il dira: « Le lien [britannique] demeure ce qu'il a toujours été, c'est-à-dire non pas matériel, mais spirituel. Il s'agit du lien d'une tradition politique commune, d'institutions politiques communes et d'une conception commune des affaires publiques » (ibid., 100).

22. Zimmern s'appuie également sur Acton (1918 [1914]; 1929).

23. «La nationalité est éminemment porteuse de coutumes, d'habitudes irraisonnées et d'idées transmises qui étouffent l'individualité [...]. La nationalité doit être abordée avec discernement. Elle n'est pas toujours libérale ou constructive. Elle peut être aussi dangereuse lorsque ses frontières débordent celles de l'État que salutaire lorsqu'elles y sont confinées » $(1917,182)$. 
24. Il applique ce terme à Dilke tout comme à Durham en raison de l'anglocentricité de leur position.

25. Dans un élan d'idéalisme, Zimmern affirmera: « Le principe britannique établi eu égard à la discrimination raciales (sic... vérifier la citation) est celui d'une égalité complète » (ibid., 111). Cf. aussi: « tout sujet britannique, quelle que soit sa race ou sa nationalité, jouit de droits égaux et ne devrait subir aucune discrimination en raison de la couleur de sa peau » (ibid.).

26. Rappelons que dans ce contexte, le roi George IV a échangé son nom de famille «Saxe-Coburg et Gotha », on ne peut plus germanique, pour le patronyme anglais de « Windsor». Le roi Albert I de Belgique, de la même famille allemande, a fait de même en 1920, adoptant alors le patronyme plus approprié de « de Belgique».

27. Le titre complet de John Buchan était: « Son Excellence le très honorable Sir John Buchan, baron Tweedsmuir, compagnon de l'ordre des compagnons d'honneur, chevalier grand-croix du très distingué ordre de Saint-Michel et SaintGeorge, chevalier grand-croix de l'ordre royal de Victoria, gouverneur général et commandant en chef de la milice et des forces navales et aériennes du Canada ».

28. Nous nous en remettrons surtout aux discours de John Buchan alors qu'il était gouverneur général du Canada, tels qu'ils apparaissent chez Buchan (1940) et Gibbon (1938). Pour ses discours non publiés, nous citerons de Henshaw (2007).

29. Pour l'adhésion de Zimmern et Buchan à ce groupe, $c f$. ibid., 52.

30. Sur le racisme de Buchan, $c f$. entre autres Magubane (1996, 238-243). Pour un bref commentaire rejetant l'idée selon laquelle Buchan aurait été antisémite, $c f$. Doidge (1989, 377).

31. Bourassa dira, avec un sens de la polémique, que « les Canadiens français ont été, en Amériques, les pionniers des institutions anglaises » (Bourassa, 1903, 21).

32. Évidemment, ce souci de l'économie et de l'éducation dans la pensée nationale canadienne-française n'est pas né avec le début du $20^{\mathrm{e}}$ siècle. Ces intuitions sont notamment anticipées par Étienne Parent (2000, 97-116; 117-143), auteur ayant influencé directement Édouard Montpetit (cf. Montpetit, 1939, introduction).

33. Le texte est aussi paru dans Montpetit (2005, 119-131). Dans la suite du texte, nous référerons aux deux éditions.

34. Sans citer nommément The Third Empire, Montpetit y fait spécifiquement référence par une allusion à la croyance de Zimmern selon laquelle l'Empire aurait manqué d'une philosophie (Montpetit, 2005, 121; 1937, 15), idée que Zimmern exprime à la p. 13 de son ouvrage.

35. $C f$. « Les trois unités » $(1937,43)$ : « Le génie de chaque groupe enrichit le dominion comme la grandeur de chaque dominion enrichit l'Empire. Le nier, c'est nier l'Empire $[\ldots] »$.

\section{Bibliographie}

Acton [John Emerich Edward Dalberg, Lord]. "Nationality" The History of Freedom and Other Essays. London: Macmillan, 1907.

-. Selections from the Correspondence of the First Lord Acton. Ed. J. N. Figgis et R. V. Laurence, vol. 1. Londres: Longmans, Greens and Co., 1917. 
Ajzenstat, Janet. "Canada's First Constitution: Pierre Bédard on Tolerance and Dissent" Canadian Journal of Political Science 23 (1990): 39-57.

—. "Celebrating 1791: Two Hundred Years of Representative Government" Canadian Parliamentary Review 14.1 (Spring 1991): 26-30.

Armitage, D. The Ideological Origins of the British Empire. Cambridge: Cambridge University Press, 2000.

Berger, Carl. Sense of Power: Studies in the Ideas of Canadian Imperialism. Toronto: University of Toronto Press, 1970.

Bibby, Reginald W. Mosaic Madness. The Poverty and Potential of Life in Canada. Toronto: Stoddart, 1990.

Bissoondath, Neil. Le marché des illusions. Montréal: Boréal, 1995.

Bock-Côté, Mathieu. La dénationalisation tranquille. Montréal: Boréal, 2007.

—. « La fabrique du multiculturalisme : le cours ECR en contexte » L'Action nationale (sept. 2009): 18-32.

Bourassa, Henri. Les Canadiens français et l'Empire britannique. Québec: Imprimerie S.-A. Demers, 1903.

- "Mr. Bourassa's Views on the Participation of Canada in the War - The Past and the Future" L'Encyclopédie de l'histoire du Québec / The Quebec History Encyclopedia. Éd. Claude Bélanger. Montréal: Marianapolis College, 2009. Le 14 décembre $2009<$ http://faculty.marianopolis.edu/c.belanger/quebechistory/ encyclopedia/Bourassanationalism.html $>$

Breton, Eric. "Canadian Federalism, Multiculturalism, and the Twenty-first Century" Journal of Canadian Studies 21 (2000): 155-175.

Buchan, John (Lord Tweedsmuir). The King's Grace, 1910-1935. London: Hodder and Stoughton, 1935.

—. Canadian Occasions. Freeport, NY: Books for Libraries Press, 1940.

Bucker, Phillip et R. Douglas Francis. Canada and the British World: Culture Migration, and Identity. Vancouver: UBC Press, 2006. 1-28.

Cannadine, David. "Imperial Canada: Old History, New Problems" Imperial Canada, 1867-1917. Ed. Colin Coates. Edinburgh: University of Edinburgh Press, 1997. 1-19.

Canny, Nicholas. The Origins of Empire. The Oxford History of the British Empire. Oxford: Oxford University Press, 1998.

Coates, Colin M. "French Canadians' Ambivalence to the Empire" Canada and the British Empire. Ed. Phillip Buckner. Oxford: Oxford University Press, 2008. 181-199.

Colley, Linda. Britons: Forging the Nation, 1707-1837. $2^{\text {nd }}$ edition. London: Pimlico, 2003.

Darwin, John. "A Third British Empire? The Dominion Idea in Imperial Politics" Oxford History of the British Empire. Ed. Judith Brown et W. Roger Louis. Vol. 4: The Twentieth Century. Oxford: Oxford University Press, 1999. 64-87.

—. "Imperial Twilight, or When Did the Empire End?" Canada and the End of Empire. Ed. Philip Buckner. Vancouver: UBC Press, 2005. 15-24.

Day, Richard J.F. Multiculturalism and the History of Canadian Diversity. Toronto: University of Toronto Press. 2000.

Dilke, Charles Wentworth. Greater Britain: A Record of Travel in English-speaking countries during 1866-1867. New York: Harper and Brothers, 1869.

—. Problems of Greater Britain. London: Macmillan, 1890. 
Doidge, Peter W. E. “John Buchan's Anti-Semitism” The Expository Times 100 (1989): 377.

Foster, Kate A. Our Canadian Mosaic. Toronto: Dominion Council, Y.W.C.A, 1926.

Fournier Marcel. «Édouard Montpetit et l'Université moderne, ou l'échec d'une génération » Revue d'histoire de l'Amérique française 36.1 (1982): 3-29.

Gagnon, Alain G. « Plaidoyer pour l'interculturalisme » Possibles 24.4 (2000): 11-25.

Gagnon, L. « Le modèle canadien » La Presse (10 novembre 2007): PLUS 4.

Gibbon, John Murray. Canadian Mosaic: The Making of a Northern Nation. Toronto: McClelland \& Stewart, 1938.

Grant, George. Lament for a Nation: the Defeat of Canadian Nationalism. Toronto: McClelland \& Stewart, 1965.

-. Est-ce la fin du Canada? Lamentation sur l'échec du nationalisme canadien. Lasalle (Qc): Hurtubise HMH, 1987.

Gwyn, R. Nationalism Without Wall: The Unbearable Lightness of Being Canadian. Toronto: McClelland and Stewart, 1995.

Hall, Hessel Duncan. The British Commonwealth of Nations. London: Methuen \& Co., 1920.

Hancock, William Keith. Survey of British Commonwealth Affairs. London: Oxford University Press, 1937.

Henshaw, Peter. "John Buchan and the British Imperial Origins of Canadian Multiculturalism" Canadas of the Mind: the Making and Unmaking of Canadian Nationalisms in the Twentieth Century. Ed. Norman Hillmer et Adam Chapnick. Montréal: McGill's University Press, 2007. 191-213.

Hillebrand, Ernst. «Atmosphère suffocante dans le Londonistan » Analyses et documents. Paris: Friedrich-Ebert-Stiftung. 2006. Le 9 janvier $2010<$ http:// www.communautarisme.net/attachment/27380/s

Holmes, Janice. "Victoria's Ireland: Britishness and Irishness, 1837-1901" History Ireland 9.4 (2001): 6-7.

Hughes, Matthew (éd.).Social Trends 39. Office for National Statistics. Basingstoke: Palgrave Macmillan, 2009. 4.

Imperial Conference. [Balfour Declaration] Imperial conference 1926. Interimperial relations committee report, proceedings and memoranda (E. 129). Le 18 novembre $2009<h t t p: / / w w w . f o u n d i n g d o c s . g o v . a u / r e s o u r c e s / t r a n s c r i p t s /$ cth11_doc_1926.pdf>

Kenny, Jason. « Notes d'allocution pour l'honorable Jason Kenney [...] lors de la cérémonie de remise du Prix Paul Yuzyk » Citoyenneté et Immigration Canada. 2009. Le 9 janvier 2010 http://www.cic.gc.ca/francais/ministere/medial discours/2009/2009-06-12.asp>

Kymlicka, Will. La citoyenneté multiculturelle. Montréal: Boréal, 2001.

—. La voie canadienne. Montréal: Boréal, 2003.

Lacombe, Sylvie. La rencontre de deux peuples élus. Comparaison des ambitions nationale et impériale au Canada entre 1896 et 1920. Sainte-Foy (Québec): Presses de l'Université Laval, 2002.

-. A Brief Overview of French Canadian Nationalism in the $19^{\text {th }}$ Century. Université Laval, 2006. Le 21 janvier $2012<$ http://www.fss.ulaval.ca/cms/ upload/soc/fichiers/lacombe.pdf>

Magubane, Bernard. The Making of a Racist State: British Imperialism and the Union of South Africa, 1875-1910. Trenton: Africa New World Press, 1996. 
Markwell, D. J. "Zimmern, Sir Alfred Eckhard (1879-1957)" Oxford Dictionary of National Biography. Ed. H. C. G. Matthew et Brian Harrison. Oxford: OUP, 2004. Le 18 novembre 2009 <http://www.oxforddnb.com.login.ezproxy.library. ualberta.ca/view/article/37088>

McIntyre, W. David. The Britannic Vision. Basingstoke: Palgrave MacMillan, 2009.

Millat, Gilbert. Le déclin de la Grande-Bretagne au XXe siècle dans le dessin de presse. Paris: L'Harmattan, 2008.

Miller, J. D. B. "The Commonwealth and World Order: the Zimmern Vision and After" Journal of Imperial and Commonwealth History 8 (1979-80): 159-74.

Montpetit, Edouard. « Notre avenir » La Revue trimestrielle canadienne 8 (1917): 305-321.

—. D'azur à trois lys d'or. Montréal: Éditions de l'ACF, 1937.

—. La conquête économique, tome 1 : Les forces essentielles. Montréal: Bernard Valiquette, 1939.

—. Réflexions sur la question nationale. Saint-Laurent: Bibliothèque québécoise, 2005.

Palmer, Howard. "Reluctant Hosts: Anglo-Canadian Views on Multiculturalism in the Twentieth Century" Readings in Canadian History, Post-Confederation. Ed.

R.D. Francis et D. B. Smith. Toronto: Holt, Rhinhart \& Winston, 1990. 192-209.

Parent, Étienne. Étienne Parent. Discours. C. Couture et Y. Lamonde (éd.).

Montréal: Presses de l'Université de Montréal (Bibliothèque du Nouveau Monde), 2000.

Quigley, Carroll. The Anglo-American Establishment. New York: Books in Focus, 1981.

Resnick, Philip. The European Roots of Canadian Identity. Peterborough: Broadview Press, 2005.

Rich, Paul. "Alfred Zimmern's Cautious Idealism: The League of Nations, International Education, and the Commonwealth". Long, D. et P. Wilson. Thinkers of the Twenty Years' Crisis: Inter-War Idealism Reassessed. Oxford: Clarendon Press, 2003. 79-99.

Saunders, Doug. "Mistaken Identity" The Globe and Mail (27 juin 2009): F1.

Severance, John B. Gandhi, Great Soul. New York: Houghton Mifflin, 1997.

Smith, Adrian. The New Statesman: Portrait of a Political Weekly, 1913-1931. London: Frank Cass, 1996.

Sosoe, L. K. Diversité humaine. Démocratie, multiculturalisme et citoyenneté. Sainte-Foy: Presses de l'Université Laval, 2002.

Stoffman, Daniel. Who Gets In: What's Wrong With Canada's Immigration Program - How To Fix It. Toronto: Macfarlane Walter \& Ross, 2002.

Taguieff, Pierre-André. La judéophobie des modernes. Des Lumières au Jihad mondial. Paris: Éditions Odile Jacob, 2008.

Taylor, Charles. "The Politics of Recognition" Multiculturalism and the Politics of Recognition. Dir. A. Gutman. Princeton: Princeton University Press, 1992.

Taylor, Martin Brook. Promoters, Patriots, and Partisans: Historiography in Nineteenth-Century English Canada. Toronto: University of Toronto Press, 1989.

Thompson, John Herd. "Canada and the 'Third British Empire', 1901-1939" Canada and the British Empire. Ed. Phillip Buckner. Oxford: Oxford University Press, 2008. 87-106.

Trudeau, Pierre Elliott. Le fédéralisme et la société canadienne-française. Montréal: HMH, 1967. 
Wardhaugh, Robert. "Gateway to Empire: Imperial Sentiment in Winnipeg, 18671917” Imperial Canada, 1867-1917. Ed. Colin Coates. Edinburgh: University of Edinburgh Press, 1997. 206-219.

Wayland, Sarah. "Immigration, Multiculturalism and National Identity in Canada" International Journal on Group Rights 5 (1997): 33-58.

Yuzyk, Paul. "Speech on Multiculturalism in Senate of Canada" Debates (1964): 50-58.

Zimmern, Alfred. "German Culture and the British Commonwealth" Nationality and Government. London: Chatto \& Windus, 1918 [1914]. 1-31.

-. "Nationalism and Internationalism". Prospects of Democracy and Other Essays. London: Chatto and Windus, 1929. 76-93.

—. The Third British Empire. $3^{\text {rd }}$ edition. Oxford: Oxford University Press, 1934. 